\title{
Generating Context-Free Grammars using Classical Planning
}

\author{
Javier Segovia-Aguas ${ }^{1}$, Sergio Jiménez ${ }^{2}$, Anders Jonsson ${ }^{1}$ \\ ${ }^{1}$ Universitat Pompeu Fabra, Barcelona, Spain \\ ${ }^{2}$ University of Melbourne, Parkville, Australia \\ javier.segovia@upf.edu,sjimenez@unimelb.edu.au, anders.jonsson@upf.edu
}

\begin{abstract}
This paper presents a novel approach for generating Context-Free Grammars (CFGs) from small sets of input strings (a single input string in some cases). Our approach is to compile this task into a classical planning problem whose solutions are sequences of actions that build and validate a CFG compliant with the input strings. In addition, we show that our compilation is suitable for implementing the two canonical tasks for CFGs, string production and string recognition.
\end{abstract}

\section{Introduction}

A formal grammar is a set of symbols and rules that describe how to form the strings of certain formal language. Usually two tasks are defined over formal grammars:

- Production: Given a formal grammar, generate strings that belong to the language represented by the grammar.

- Recognition (also known as parsing): Given a formal grammar and a string, determine whether the string belongs to the language represented by the grammar.

Chomsky defined four classes of formal grammars that differ in the form and generative capacity of their rules [Chomsky, 2002]. In this paper we focus on Context-Free Grammars (CFGs), where the left-hand side of a grammar rule is always a single non-terminal symbol. This means that the symbols on the left-hand side of CFG rules do not appear in the strings that belong to the corresponding language.

To illustrate this Figure 1(a) shows an example CFG that contains a single non-terminal symbol, $S$, and three terminal symbols ( $a, b$ and $\epsilon$, where $\epsilon$ denotes the empty string). This CFG defines three production rules that can generate, for instance, the string aabbaa by applying the first rule twice, then the second rule once and finally, the third rule once again. The parse tree in Figure 1(b) exemplifies the previous rule application and proves that the string aabbaa belongs to the language defined by the grammar.

Learning the entire class of CFGs using only positive examples, i.e. strings that belong to the corresponding formal language, is not identifiable in the limit [Gold, 1967]. In this paper we address the generation of CFGs from positive examples but: (1) for a bounded maximum number of non-terminal

$$
\begin{aligned}
& S \rightarrow a S a \\
& S \rightarrow b S b \\
& S \rightarrow \epsilon
\end{aligned}
$$

$S$

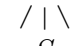

a $S$ a

$/ 11$

a $S$ a

$/ 1 \backslash$

$b S b$

।

(a)

(b)

Figure 1: (a) Example of a context-free grammar; (b) the corresponding parse tree for the string aabbaa.

symbols in the grammar and (2), a bounded maximum size of the rules in the grammar (i.e. a maximum number of symbols in the right-hand side of the grammar rules).

Our approach is compiling this inductive learning task into a classical planning task whose solutions are sequences of actions that build and validate a CFG compliant with the input strings. The reported empirical results show that our compilation can generate CFGs from small amounts of input data (even a single input string in some cases) using an off-theshelf classical planner. In addition, we show that the compilation is also suitable for implementing the two canonical tasks of CFGs, string production and string recognition.

\section{Background}

This section defines the formalization of CFGs and the planning models that we adopt in this work.

\subsection{Context-Free Grammars}

We define a CFG as a tuple $\mathcal{G}=\left\langle V, v_{0}, \Sigma, R\right\rangle$ where:

- $V$ is the finite set of non-terminal symbols, also called variables. Each $v \in V$ represents a sub-language of the language defined by the grammar.

- $v_{0} \in V$ is the start non-terminal symbol that represents the whole grammar.

- $\Sigma$ is the finite set of terminal symbols, which are disjoint from the set of non-terminal symbols, i.e. $V \cap \Sigma \neq \emptyset$. The set of terminal symbols is the alphabet of the language defined by $\mathcal{G}$ and can contain the empty string, which we denote by $\epsilon \in \Sigma$. 
- $R: V \rightarrow(V \cup \Sigma)^{*}$ is the finite set of production rules in the grammar. By definition rules $r \in R$ always contain a single non-terminal symbol on the left-hand side.

Figure 1(a) shows a 1-variable CFG since it defines a single non-terminal symbol. In this example $V=\{S\}, v_{0}=S$, $\Sigma=\{a, b, \epsilon\}$, and $R=\{S \rightarrow a S a, S \rightarrow b S b, S \rightarrow \epsilon\}$.

For any two strings $e_{1}, e_{2} \in(V \cup \Sigma)^{*}$ we say that $e_{1} d i$ rectly yields $e_{2}$, denoted by $e_{1} \Rightarrow e_{2}$, iff there exists a rule $\alpha \rightarrow \beta \in R$ such that $e_{1}=u_{1} \alpha u_{2}$ and $e_{2}=u_{1} \beta u_{2}$ with $u_{1}, u_{2} \in(V \cup \Sigma)^{*}$. Furthermore we say $e_{1}$ yields $e_{2}$, denoted by $e_{1} \Rightarrow^{*} e_{2}$, iff $\exists k \geq 0$ and $\exists u_{1}, \ldots, u_{k}$ such that $e_{1}=u_{1} \Rightarrow u_{2} \Rightarrow \ldots \Rightarrow u_{k}=e_{2}$. For instance, Figure 1(b) shows how the string $S$ yields the string aabbaa. The language of a CFG, $L(\mathcal{G})=\left\{e \in \Sigma^{*}: v_{0} \Rightarrow^{*} e\right\}$, is the set of strings that contain only terminal symbols and that can be yielded from the string that contains only the initial non-terminal symbol $v_{0}$.

Given a CFG $\mathcal{G}$ and a string $e \in L(\mathcal{G})$ that belongs to its language, we define a parse tree $t_{\mathcal{G}, e}$ as an ordered, rooted tree that determines a concrete syntactic structure of $e$ according to the rules in $\mathcal{G}$ :

- Each node in a parse tree $t_{\mathcal{G}, e}$ is either:

- An internal node that corresponds to the application of a rule $r \in R$.

- A leaf node that corresponds to a terminal symbol $\sigma \in \Sigma$ and has no outgoing branches.

- Edges in a parse tree $t_{\mathcal{G}, e}$ connect non-terminal symbols to terminal or non-terminal symbols following the rules $R$ in $\mathcal{G}$.

\subsection{Classical Planning with Conditional Effects}

We use the model of classical planning with conditional effects because conditional effects allow to adapt the execution of a sequence of actions to different initial states, as shown in conformant planning [Palacios and Geffner, 2009]. The support of conditional effects is now a requirement of the International Planning Competition [Vallati et al., 2015] and most current classical planners natively cope with conditional effects without compiling them away [Helmert, 2006].

We use $F$ to denote a set of propositional variables or $f u$ ents describing a state. A literal $l$ is a valuation of a fluent $f \in F$, i.e. $l=f$ or $l=\neg f$. A set of literals $L$ on $F$ represents a partial assignment of values to fluents (WLOG we assume that $L$ does not assign conflicting values to any fluent). We use $\mathcal{L}(F)$ to denote the set of all literal sets on $F$, i.e. all partial assignments of values to fluents. Given $L$, let $\neg L=\{\neg l: l \in L\}$ be the complement of $L$.

A state $s$ is a set of literals such that $|s|=|F|$, i.e. a total assignment of values to fluents. The number of states is then $2^{|F|}$. Explicitly including negative literals $\neg f$ in states simplifies subsequent definitions, but we often abuse notation by defining a state $s$ only in terms of the fluents that are true in $s$, as is common in STRIPS planning.

A classical planning frame is a tuple $\Phi=\langle F, A\rangle$, where $F$ is a set of fluents and $A$ is a set of actions with conditional effects. Each action $a \in A$ has a set of literals $\operatorname{pre}(a) \in$ $\mathcal{L}(F)$ called the precondition and a set of conditional effects cond $(a)$. Each conditional effect $C \triangleright E \in \operatorname{cond}(a)$ is composed of two sets of literals $C \in \mathcal{L}(F)$ (the condition) and $E \in \mathcal{L}(F)$ (the effect).

An action $a \in A$ is applicable in state $s$ if and only if $\operatorname{pre}(a) \subseteq s$, and the resulting set of triggered effects is

$$
\operatorname{eff}(s, a)=\bigcup_{C \triangleright E \in \operatorname{cond}(a), C \subseteq s} E,
$$

i.e. effects whose conditions hold in $s$. The result of applying $a$ in $s$ is a new state $\theta(s, a)=(s \backslash \neg \operatorname{eff}(s, a)) \cup \operatorname{eff}(s, a)$.

Given a frame $\Phi=\langle F, A\rangle$, a classical planning instance is a tuple $P=\langle F, A, I, G\rangle$, where $I \in \mathcal{L}(F)$ is an initial state (i.e. $|I|=|F|$ ) and $G \in \mathcal{L}(F)$ is a goal condition. We consider the fragment of classical planning with conditional effects that includes negative conditions and goals.

A plan for $P$ is an action sequence $\pi=\left\langle a_{1}, \ldots, a_{n}\right\rangle$ that induces a state sequence $\left\langle s_{0}, s_{1}, \ldots, s_{n}\right\rangle$ such that $s_{0}=I$ and, for each $i$ such that $1 \leq i \leq n, a_{i}$ is applicable in $s_{i-1}$ and generates the successor state $s_{i}=\theta\left(s_{i-1}, a_{i}\right)$. The plan $\pi$ solves $P$ if and only if $G \subseteq s_{n}$, i.e. if the goal condition is satisfied following the application of $\pi$ in $I$.

\subsection{Generalized Planning}

Our approach for learning CFGs from positive examples is to model this task as a generalized planning problem that is eventually solved with an off-the-shelf classical planner using the compilation proposed by Jiménez and Jonsson (2015).

We define a generalized planning problem as a finite set of classical planning instances $\mathcal{P}=\left\{P_{1}, \ldots, P_{T}\right\}$ within the same planning frame $\Phi$. Therefore, $P_{1}=$ $\left\langle F, A, I_{1}, G_{1}\right\rangle, \ldots, P_{T}=\left\langle F, A, I_{T}, G_{T}\right\rangle$ share the same fluents and actions and differ only in the initial state and goals. Although actions are shared, each action can have different interpretations in different states due to conditional effects. Given a planning frame $\Phi=\langle F, A\rangle$, then $\Gamma(\Phi)=$ $\{\langle F, A, I, G\rangle: I \in \mathcal{L}(F),|I|=|F|, G \in \mathcal{L}(F)\}$ denotes the set of all planning instances that can be instantiated from $\Phi$ by defining an initial state $I$ and a goal condition $G$.

A solution $\Pi$ to a generalized planning problem $\mathcal{P}$ is a generalized plan that solves every individual instance $P_{t}$, $1 \leq t \leq T$. Generalized plans may have diverse forms that range from DS-planners [Winner and Veloso, 2003] and generalized polices [Martín and Geffner, 2004] to finite state machines [Bonet et al., 2010; Segovia-Aguas et al., 2016b], AND/OR graphs or CFGs [Ramirez and Geffner, 2016].

In this work we assume that the solution to a generalized planning problem $\mathcal{P}$ is in the form of a planning program [Jiménez and Jonsson, 2015; Segovia-Aguas et al., 2016a]. Briefly, a planning program is a set of procedures, each a sequence of planning actions enhanced with goto instructions and procedure calls. However, in this work there is no need to include goto instructions since they are not needed to represent CFGs.

Given a frame $\Phi=\langle F, A\rangle$, a planning program with procedures is a finite set of planning programs $\Pi=\left\{\Pi_{0}, \ldots, \Pi_{m}\right\}$ (we adopt the convention of designating $\Pi_{0}$ as the main program, and $\left\{\Pi_{1}, \ldots, \Pi_{m}\right\}$ as the auxiliary procedures). Every $\Pi_{j}, 0 \leq j \leq m$ is then a sequence of instructions 


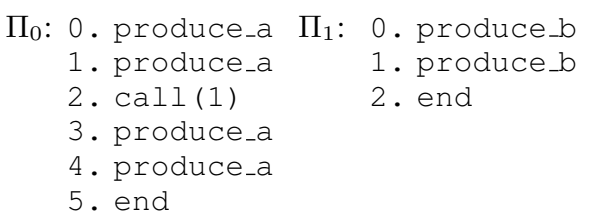

Figure 2: Example of a planning program $\Pi=\left\{\Pi_{0}, \Pi_{1}\right\}$ with one auxiliary procedure that produces the string aabbaa.

$\Pi_{j}=\left\langle w_{0}, \ldots, w_{n}\right\rangle$. Each instruction $w_{i}, 0 \leq i \leq n$, is associated with a program line $i$ and is drawn from the instructions set $\mathcal{I}=A \cup \mathcal{I}_{\text {call }} \cup\{$ end $\}$, where $\mathcal{I}_{\text {call }}=$ $\left\{\operatorname{call}\left(j^{\prime}\right): 0 \leq j^{\prime} \leq m\right\}$ is the set of procedure calls, allowing any procedure to call any other procedure on an arbitrary program line $\left(j=j^{\prime}\right.$ implies a recursive call). Figure 2 shows an example of a planning program with one auxiliary procedure that produces the string aabbaa. In this example, $A=\{$ produce_a, produce_b $\}$, where produce_a has the effect of producing the terminal symbol a.

To define the execution model of a planning program with procedures we first introduce the notion of a call stack that keeps track of where control should return when the execution of a procedure terminates. Each element of the call stack is a tuple $(j, i)$, where $j$ is an index that refers to a procedure $\Pi_{j}$, $0 \leq j \leq m$ and $i$ is a program line, $0 \leq i \leq\left|\Pi_{j}\right|$. In what follows we use $\Omega \oplus(j, i)$ to denote a call stack recursively defined by a call stack $\Omega$ and a top element $(j, i)$.

The execution model for a planning program $\Pi$ consists of a program state $(s, \Omega)$, where $s$ is a planning state and $\Omega$ is a call stack. Given a program state $(s, \Omega \oplus(j, i))$, the execution of instruction $w_{i}^{j}$ on line $i$ of procedure $\Pi_{j}$ is defined as:

- If $w_{i}^{j} \in A$, the new program state is $\left(s^{\prime}, \Omega \oplus(j, i+1)\right)$, where $s^{\prime}=\theta\left(s, w_{i}^{j}\right)$ is the state resulting from applying action $w_{i}^{j}$ in state $s$.

- If $w_{i}^{j}=\operatorname{call}\left(j^{\prime}\right)$, the new program state is $(s, \Omega \oplus$ $\left.(j, i+1) \oplus\left(j^{\prime}, 0\right)\right)$. In other words, calling a procedure $\Pi_{j^{\prime}}$ has the effect of (1) incrementing the program line at the top of the stack; and (2) pushing a new element onto the call stack to start the execution of $\Pi_{j^{\prime}}$ on line 0 .

- If $w_{i}^{j}=$ end, the new program state is $(s, \Omega)$, i.e. has the effect of terminating a procedure by popping element $(j, i)$ from the top of the call stack.

The execution of a planning program with procedures terminates when executing an end instruction empties the call stack, i.e. in program states $(s, \emptyset)$.

To execute a planning program with procedures $\Pi$ on a planning problem $P=\langle F, A, I, G\rangle$, we set the initial program state to $(I,(0,0))$, i.e. execution is in the initial planning state, on program line 0 of the main program $\Pi_{0}$. To ensure that the execution model remains bounded we impose an upper bound $\ell$ on the size of the call stack.

Executing $\Pi$ on $P$ can fail for any of these four reasons:

1. Execution terminates in program state $(s, \emptyset)$ but the goal condition does not hold, i.e. $G \nsubseteq s$.
2. When executing action $w_{i} \in A$ in program state $(s, \Omega)$, the precondition of $w_{i}$ does not hold, i.e. $\operatorname{pre}\left(w_{i}\right) \not s$.

3. Execution enters an infinite loop that never reaches a program state $(s, \emptyset)$.

4. Executing a call $\left(j^{\prime}\right)$ instruction in program state $(s, \Omega)$ exceeds the stack size $|\Omega|=\ell$, i.e. causes a stack overflow.

We say that $\Pi$ solves $P$ iff the goal condition holds when the execution of $\Pi$ on $P$ terminates, i.e. $(s, \emptyset) \wedge G \subseteq s$. Given a generalized planning problem $\mathcal{P}=\left\{P_{1}, \ldots, P_{T}\right\}$, a planning program $\Pi$ solves $\mathcal{P}$ iff $\Pi$ solves each planning instance $P_{t}, 1 \leq t \leq T$.

\subsection{Computing Planning Programs}

A classical planner can detect the four failure conditions defined above, making it particularly suitable for generating planning programs. We briefly describe here the compilation from generalized planning to classical planning for computing planning programs [Jiménez and Jonsson, 2015; Segovia-Aguas et al., 2016a].

Given a generalized planning problem $\mathcal{P}=\left\{P_{1}, \ldots, P_{T}\right\}$, the bounds on the number of program lines $n$, auxiliary procedures $m$ and stack size $\ell$, the compilation outputs a classical planning problem $P_{n, m}^{\ell}=\left\langle F_{n, m}^{\ell}, A_{n, m}^{\ell}, I_{n, m}^{\ell}, G_{n, m}^{\ell}\right\rangle$ such that any classical plan $\pi$ that solves $P_{n, m}^{\ell}$ programs the instructions of a planning program $\Pi$ and simulates the execution of $\Pi$ on each $P_{1<t<T}$, validating that $\Pi$ solves $\mathcal{P}$.

In more detail, the set of fluents $F_{n, m}^{\ell}$ extends $F$ with:

- $F_{p c}^{\ell}=\left\{\mathrm{pc}_{i}^{k}: 0 \leq i \leq n, 1 \leq k \leq \ell\right\} \cup\left\{\operatorname{proc}_{j}^{k}: 0 \leq\right.$ $j \leq m, 1 \leq k \leq \ell\}$ representing the current line and procedure.

- $F_{\text {top }}^{\ell}=\left\{\text { top }^{k}\right\}_{0 \leq k \leq \ell}$ representing the top level of the stack at the current time.

- $F_{i n s}^{m}$ encoding the instructions in the main and auxiliary procedures, which is formally defined as $F_{i n s}^{m}=$ $\left\{\right.$ ins $_{i, j, w}: 0 \leq i \leq n, 0 \leq j \leq m, w \in A \cup \mathcal{I}_{\text {call }} \cup$ $\{$ nil, end $\}$, where nil indicates that the instruction on line $i$ of procedure $j$ is empty, i.e. can be programmed.

- done, a fluent marking we are done programming and executing the planning program.

$A_{n, m}^{\ell}$ defines the following actions:

- An action $w_{i, j}^{k}$ for each action instruction $w \in A$, program line $i$, procedure $j$ and stack level $k$ :

$$
\begin{aligned}
\operatorname{pre}\left(w_{i, j}^{k}\right) & =\operatorname{pre}(w) \cup\left\{\mathrm{pc}_{i}^{k}, \operatorname{top}^{k}, \operatorname{proc}_{j}^{k}\right\}, \\
\operatorname{cond}\left(w_{i, j}^{k}\right) & =\operatorname{cond}(w) \cup\left\{\emptyset \triangleright\left\{\neg \mathrm{pc}_{i}^{k}, \mathrm{pc}_{i+1}^{k}\right\}\right\} .
\end{aligned}
$$

- An action call $j_{i, j}^{j^{\prime}, k}$ for each $\operatorname{call}\left(j^{\prime}\right) \in \mathcal{I}_{\text {call }}$ that pushes a new program line $\left(j^{\prime}, 0\right)$ onto the stack:

$$
\begin{aligned}
\operatorname{pre}\left(\mathrm{call}_{i, j}^{j^{\prime}, k}\right)= & \left\{\mathrm{pc}_{i}^{k}, \operatorname{top}^{k}, \operatorname{proc}_{j}^{k}\right\}, \\
\operatorname{cond}\left(\mathrm{call}_{i, j}^{j^{\prime}, k}\right)= & \left\{\emptyset \triangleright \left\{\neg \mathrm{pc}_{i}^{k}, \mathrm{pc}_{i+1}^{k}, \neg \text { top }^{k}, \text { top }^{k+1},\right.\right. \\
& \left.\left.\mathrm{pc}_{0}^{k+1}, \operatorname{proc}_{j^{\prime}}^{k+1}\right\}\right\} .
\end{aligned}
$$


- Actions end $d_{i, j}^{k+1}$ that simulate the termination on line $i$ of procedure $j$ on stack level $k+1,0 \leq k<\ell$ :

$$
\begin{aligned}
\operatorname{pre}\left(\text { end }_{i, j}^{k+1}\right)= & \left\{\mathrm{pc}_{i}^{k+1}, \operatorname{top}^{k+1}, \operatorname{proc}_{j}^{k+1}\right\}, \\
\operatorname{cond}\left(\text { end }_{i, j}^{k+1}\right)= & \left\{\emptyset \triangleright \left\{\neg \mathrm{pc}_{i}^{k+1}, \neg \operatorname{top}^{k+1}, \neg \operatorname{proc}_{j}^{k+1},\right.\right. \\
& \left.\left.\operatorname{top}^{k}\right\}\right\} .
\end{aligned}
$$

The effect is to pop the program line $(j, i)$ from the top of the stack.

The action set $A_{n, m}^{\ell}$ contains the following actions:

- For each instruction $w_{i, j}^{k}, w \in A \cup \mathcal{I}_{\text {call }} \cup\{$ end $\}$, an action $P\left(w_{i, j}^{k}\right)$ that programs $w$, and a repeat action $R\left(w_{i, j}^{k}\right)$ that executes $w$ when already programmed:

$$
\begin{aligned}
\operatorname{pre}\left(P\left(w_{i, j}^{k}\right)\right) & =\operatorname{pre}\left(w_{i, j}^{k}\right) \cup\left\{\text { ins }_{i, j, \text { nil }}\right\}, \\
\operatorname{cond}\left(P\left(w_{i, j}^{k}\right)\right) & =\left\{\emptyset \triangleright\left\{\text { ins }_{i, j, \text { nil }}, \text { ins }_{i, j, w}\right\}\right\}, \\
\operatorname{pre}\left(R\left(w_{i, j}^{k}\right)\right) & =\operatorname{pre}\left(w_{i, j}^{k}\right) \cup\left\{\text { ins }_{i, j, w}\right\}, \\
\operatorname{cond}\left(R\left(w_{i, j}^{k}\right)\right) & =\operatorname{cond}\left(w_{i, j}^{k}\right) .
\end{aligned}
$$

- For each planning problem $P_{t}, 1 \leq t \leq T$, a termination action term $\mathrm{t}_{t}$ that simulates the successful termination of the planning program on $P_{t}$ when the stack is empty:

$$
\begin{aligned}
\operatorname{pre}\left(\operatorname{term}_{t}\right)= & G_{t} \cup\left\{\operatorname{top}^{0}\right\}, t<T, \\
\operatorname{cond}\left(\operatorname{term}_{t}\right)= & \left\{\emptyset \triangleright\left\{\neg \operatorname{top}^{0}, \operatorname{top}^{1}, \operatorname{pc}_{0}^{1}, \operatorname{proc}_{0}^{1}\right\}\right\} \cup \\
& \left\{\{\neg f\} \triangleright\{f\}: f \in I_{t+1}\right\} \cup \\
& \left\{\{f\} \triangleright\{\neg f\}: f \notin I_{t+1}\right\}, t<T, \\
\operatorname{pre}\left(\operatorname{term}_{T}\right)= & G_{T} \cup\left\{\operatorname{top}^{0}\right\}, \\
\operatorname{cond}\left(\operatorname{term}_{T}\right)= & \{\emptyset \triangleright\{\text { done }\}\} .
\end{aligned}
$$

Note that the effect of term ${ }_{t}, t<T$, is to reset the program state to the initial state of problem $P_{t+1}$.

The initial state sets all the program lines (main and auxiliary procedures) as empty and sets the procedure on stack level 1 to $\Pi_{0}$ (the main procedure) with the program counter pointing to the first line of that procedure. The initial state on fluents in $F$ is $I_{1}$, hence $I_{n, m}^{\ell}=I_{1} \cup\left\{\right.$ ins $_{i, j, \text { nil }}: 0 \leq i \leq$ $n, 0 \leq j \leq m\} \cup\left\{\right.$ top $^{1}, \mathrm{pc}_{0}^{1}$, $\left.\operatorname{proc}_{0}^{1}\right\}$. The goal condition is defined as $G_{n, m}^{\ell}=\{$ done $\}$.

\section{Generating Context-Free Grammars}

This section explains our approach to generating CFGs from input strings using classical planning. We formalize this task as a tuple $\langle\Sigma, E, m\rangle$, where:

- $\Sigma$ is the finite set of terminal symbols.

- $E=\left\{e_{1}, \ldots, e_{T}\right\}$ is the finite set of input strings containing only terminal symbols: $e_{t} \in \Sigma^{*}, 1 \leq t \leq T$.

- $m$ is a bound on the number of non-terminal symbols $V_{m}=\left\{v_{0}, \ldots, v_{m}\right\}$. As a consequence, $m$ implicitly defines the space of possible rules, $V_{m} \rightarrow\left(V_{m} \cup \Sigma\right)^{*}$.

A solution to this inductive learning task is a CFG $\mathcal{G}=$ $\left\langle V_{m}, v_{0}, \Sigma, R_{m}\right\rangle$ such that, for every $e \in E$, there exists a parse tree $t_{\mathcal{G}, e}$.

$$
\begin{aligned}
& \Pi_{0}: 0 \text {. choose }(1|5| 8) \\
& \text { 1. parse_a } \\
& \text { 2. call (0) } \\
& \text { 3. parse_a } \\
& \text { 4. end } \\
& \text { 5. parse_b } \\
& \text { 6. } \operatorname{call}(0) \\
& \text { 7. parse_b } \\
& 8 \text {. end }
\end{aligned}
$$

Figure 3: Planning program that represents the CFG in Figure 1(a).

\subsection{CFG Generation as Generalized Planning}

Our approach for solving $\langle\Sigma, E, m\rangle$ is modeling this task as a generalized planning task $\mathcal{P}=\left\{P_{1}, \ldots, P_{T}\right\}$ where each input string $e_{t} \in E$ corresponds to an individual classical planning task $P_{t} \in \mathcal{P}$ such that $1 \leq t \leq T$ and $P_{t}=\left\langle F, A, I_{t}, G_{t}\right\rangle$ is defined as follows:

- $F$ comprises the fluents for modeling input strings as lists of symbols. These fluents are string $($ id, $\sigma$ ) and next(id, id2), where $0 \leq i d, i d 2 \leq z, \sigma \in \Sigma$ and $z$ is a bound on the string length. For instance, the string $a b b a$ is encoded as:

$$
\begin{aligned}
& \text { string(i0, a), string(i1, b), string(i2, b), string(i3, a), } \\
& \operatorname{next}(i 0, i 1), \operatorname{next}(i 1, i 2), \operatorname{next}(i 2, i 3), \operatorname{next}(i 3, i 4) .
\end{aligned}
$$

In addition, $F$ includes fluents $\operatorname{pos}($ id), $0 \leq i d \leq z$, to indicate the current string position, and $\operatorname{sym} \mathrm{b}(\sigma), \sigma \in \Sigma$, to indicate the symbol at the current string position.

- A contains the actions for parsing the current symbol of an input string. There is a parse ${ }_{\sigma}$ action for each symbol $\sigma \in \Sigma$, e.g. $A=\left\{\right.$ parse $_{\mathrm{a}}$, parse $\left.\mathrm{b}_{\mathrm{b}}\right\}$ for the $\mathrm{CFG}$ of Figure 1(a). Action parse e recognizes that $\sigma$ is at the current position of the string and advances the position.

$$
\begin{aligned}
& \operatorname{pre}\left(\operatorname{parse}_{\sigma}\right)=\{\operatorname{symb}(\sigma)\}, \\
& \operatorname{cond}\left(\operatorname{parse} e_{\sigma}\right)=\left\{\left\{\operatorname{pos}\left(\mathrm{i}_{1}\right), \operatorname{next}\left(\mathrm{i}_{1}, \mathrm{i}_{2}\right), \operatorname{string}\left(\mathrm{i}_{2}, \sigma^{\prime}\right)\right\} \triangleright\right. \\
& \left.\left\{\neg \operatorname{pos}\left(\mathrm{i}_{1}\right), \operatorname{pos}\left(\mathrm{i}_{2}\right), \neg \operatorname{symb}(\sigma), \operatorname{symb}\left(\sigma^{\prime}\right)\right\}: \forall \mathrm{i}_{1}, \mathrm{i}_{2}, \sigma^{\prime}\right\} .
\end{aligned}
$$

- $I_{t}$ contains the fluents encoding the $t$-th string, $e_{t} \in E$, and its initial position $\operatorname{pos}(0)$.

- $G_{t}$ requires that $e_{t}$ is parsed, i.e. $G_{t}=\left\{\operatorname{pos}\left(\left|e_{t}\right|\right)\right\}$.

According to this definition, a solution to a generalized planning problem $\mathcal{P}$ that models a CFG generation task $\langle\Sigma, E, m\rangle$ parses every $e_{t} \in E$.

\subsection{Computing CFGs with Classical Planning}

To compute CFGs with classical planning we first extend the planning program formalism, so that it can represent CFGs. Then we adapt the generalized planning compilation for computing the extended planning programs with an off-the-shelf classical planner.

Our extension of the planning program formalism augments its instruction set $\mathcal{I}$ with choice instructions. Choice instructions are intended to jump to a target line of a planning program and are defined as $\mathcal{I}_{\text {choice }}=\{$ choose $($ Target $)\}$, where Target $\subseteq\{1, \ldots, n\}$ is a subset of possible target 
program lines. Figure 3 shows a planning program with a choice instruction that encodes the CFG in Figure 1(a). In this example instruction choose $(1|5| 8)$ represents a jump to one of these three possible targets, line 1 , line 5 or line 8 .

The execution model for planning programs with choice instructions behaves as explained in Section 2.3. The conditions for termination and success are the same as in the original planning program formalism. The only new behavior that has to be defined is the execution of choice instructions. The execution of an instruction $w_{i}^{j}=$ choose(Target) on line $i$ of procedure $\Pi_{j}$ actively chooses to jump to a new line $i^{\prime} \in$ Target, changing the program state from $(s, \Omega \oplus(j, i))$ to $\left(s, \Omega \oplus\left(j, i^{\prime}\right)\right)$.

The representation of CFGs with planning programs is done associating each non-terminal symbol $v_{j} \in V_{m}$ with a planning program $\Pi_{j}$. Choice instructions always appear on the first line of $\Pi_{j}$ and represent possible jumps to the lines coding the grammar rules, $v_{j} \rightarrow\left(v_{j} \cup \Sigma\right)^{*}$, associated to the corresponding non-terminal symbol. Initially, the subset of target program lines only includes 1 and $n$, i.e. choose $(1 \mid 8)$ for the example in Figure 3. Whenever we program an end instruction on a line $i$, we add $i+1$ to the subset of target lines, leading to the choice instruction choose $(1|5| 8)$ in Figure 3.

The compilation takes as input a CFG generation task $\langle\Sigma, E, m\rangle$ such that $\left|e_{t}\right| \leq z$ for each $e_{t} \in E$, a number of program lines $n$ and a stack size $\ell$, and outputs a classical planning instance $P_{n, m}^{\ell, z}=\left\langle F_{n, m}^{\ell, z}, A_{n, m}^{\ell, z}, I_{n, m}^{\ell, z}, G_{n, m}^{\ell, z}\right\rangle$. The compilation is almost identical to the compilation described in the previous section; the only relevant difference is that $A_{n, m}^{\ell, z}$ includes actions for simulating the execution of choice instructions choose (Target) ${ }_{i^{\prime}, j}^{k}$, where $i^{\prime} \in$ Target:

$$
\begin{aligned}
& \operatorname{pre}\left(\text { choose }(\text { Target })_{i^{\prime}, j}^{k}\right)=\left\{\mathrm{pc}_{0}^{k}, \text { top }^{k}, \operatorname{proc}_{j}^{k}, \text { ins }_{0, j, \text { choose }}\right\}, \\
& \text { cond }\left(\text { choose }(\text { Target })_{i^{\prime}, j}^{k}\right)=\left\{\emptyset \triangleright\left\{\neg \mathrm{pc}_{0}^{k}, \mathrm{pc}_{i^{\prime}}^{k}\right\}\right\} \text {. }
\end{aligned}
$$

Lemma 1. Any classical plan $\pi$ that solves $P_{n, m}^{\ell, z}$ induces a valid model $\mathcal{G}=\left\langle V_{m}, v_{0}, \Sigma, R_{m}\right\rangle$ for the $C F G$ generation task $\langle\Sigma, E, m\rangle$.

Proof sketch. Once the instructions of a planning program $\Pi=\left\{\Pi_{0}, \ldots, \Pi_{m}\right\}$ are programmed they can only be executed. The classical plan $\pi$ has to program the instructions (if not yet programmed) of $\Pi$ and simulate its execution, actively choosing the jumps defined by the choice instructions and their corresponding subsets of target lines. This simulation is done for the planning task $P_{t}$ encoding the $t$-th string in $E$ and the active choice corresponds exactly to the construction of the parse tree for the $t$-th string. If this is done for every $1 \leq t \leq T$, the CFG induced by $\pi$ satisfies the solution condition for the solutions of the CFG generation task $\langle\Sigma, E, m\rangle$.

\subsection{Parsing and Production with Planning}

String production and string parsing for arbitrary CFGs can also be addressed using our compilation and an off-the-shelf classical planner.

Parsing. Let $e \notin E$ be a new string, and let $P_{e}=$ $\left\langle F_{n, m}^{\ell, z}, A_{n, m}^{\ell, z}, I_{e}, G_{e}\right\rangle$ be the classical planning instance for parsing the string $e$, i.e. instantiated on the planning frame as the problem $P_{n, m}^{\ell, z}$. In this case we can use a classical planner to determine whether $e \in L(\mathcal{G})$.

Our approach is to specify $\mathcal{G}$ in the initial state of $P_{e}$, making initially true the fluents ins $s_{i, j, w}$ that correspond to the planning program that encodes $\mathcal{G}$, and ignoring the actions $P\left(w_{i, j}^{k}\right)$ for programming instructions. A solution plan $\pi_{e}$ to $P_{e}$ is then constrained to the actions that execute the instructions specified by $\mathcal{G}$ and represents a parse tree $t_{\mathcal{G}, e}$. Essentially, parsing consists in correctly choosing the target program line $i^{\prime}$ each time a choice instruction is executed.

Interestingly parsing can also be understood as activity recognition using plan libraries that are in the form of CFGs [Ramirez and Geffner, 2016].

Production. We can produce a string $e \in L(\mathcal{G})$ using our compilation and an off-the-shelf classical planner.

Again we use a classical planning instance $P_{e}$ that represents the parsing of example $e$ with $\mathcal{G}$ also specified in the initial state of $P_{e}$ and ignoring the actions for programming instructions. The difference with the previous task is that here the linked list that encodes the string $e$ is initially empty (the corresponding fluents are false at $I_{e}$ ). This list is filled up, symbol by symbol, by the actions that execute $\mathcal{G}$ until reaching the end of the string. To do so actions parse ${ }_{\sigma}$ are replaced with actions produce ${ }_{\sigma}$ that add symbol $\sigma \in \Sigma$ at the current position of the string. Formally, produce ${ }_{\sigma}$ is defined as:

$$
\begin{aligned}
& \operatorname{pre}\left(\operatorname{produce}_{\sigma}\right)=\{\text { active }\}, \\
& \left.\operatorname{cond}(\operatorname{produce})_{\sigma}\right)=\left\{\left\{\operatorname{pos}\left(\mathrm{i}_{1}\right)\right\} \triangleright\left\{\operatorname{string}\left(\mathrm{i}_{1}, \sigma\right)\right\}: \forall \mathrm{i}_{1}\right\} \\
& \cup\left\{\left\{\operatorname{pos}\left(\mathrm{i}_{1}\right), \operatorname{next}\left(\mathrm{i}_{1}, \mathrm{i}_{2}\right)\right\} \triangleright\left\{\neg \operatorname{pos}\left(\mathrm{i}_{1}\right), \operatorname{pos}\left(\mathrm{i}_{2}\right)\right\}: \forall \mathrm{i}_{1}, \mathrm{i}_{2}\right\} \\
& \cup\left\{\left\{\operatorname{pos}\left(\mathrm{i}_{1}\right), \operatorname{next}\left(\mathrm{i}_{1}, z\right)\right\} \triangleright\{\neg \operatorname{active}\}: \forall \mathrm{i}_{1}, \mathrm{i}_{2}\right\},
\end{aligned}
$$

where active is a fluent that keeps track of whether we have reached the end of the string to be generated.

Text production using grammars and classical planning is a well studied task [Schwenger et al., 2016; Koller and Hoffmann, 2010]. This task is also related to the compilation of domain-specific control knowledge for planning [Baier et al., 2007; Alford et al., 2009].

\section{Evaluation}

We designed two types of experiments: (1) Generation, for computing CFGs compliant with a set of input strings and (2), Recognition for parsing unseen input strings given a CFG. All the experiments are run on an Intel Core i5 $3.10 \mathrm{GHz}$ x 4 with 4 GB of RAM, using the classical planner Fast Downward [Helmert, 2006], with the LAMA-2011 configuration, and a planning time limit of 600 seconds.

We created six domains that correspond to CFGs with different structure and alphabet. The domain AnBn corresponds to the $A^{n} B^{n}$ language. The Parenthesis domain corresponds to the strings that can be formed following one of two wellbalanced parenthesis distributions, sequential ()()$\ldots$ or enclosing $((\ldots))$. Parenthesis Multiple corresponds to the enclosing well-balanced parenthesis distribution but using a larger alphabet, $\Sigma=\{(,\{,[]\})$,$\} . In the Binary Arith-$ metics domain the alphabet contains two possible binary values $\{0,1\}$ and two operators $\{+,-\}$, and corresponds to the 
language of the arithmetic expressions composed by an arbitrary binary number, an operator and another binary number. For Arithmetics the alphabet includes the values $\{0, \ldots, 9\}$ and the operators $\{+,-, *, /\}$, and the language is the set of expressions formed as decimal number, operator and decimal number. Finally, a simplified English Grammar that includes Sentence, Noun Phrase and Verb Phrase as nonterminal symbols while adjective, adverb, noun and verb are terminal symbols.

Table 1 shows the results of the Generation tasks. For each domain we report the number $m$ of non-terminal symbols, the size of the stack, the procedure lines (per non-terminal symbol), the number of input strings (per non-terminal symbol) and the planning time for computing each procedure corresponding to a non-terminal symbol.

\begin{tabular}{llcrrr} 
& m & Stack & Lines & Strings & Time(s) \\
\hline AnBn & 1 & 5 & 5 & 1 & 0.3 \\
Parenthesis & 1 & 5 & 5 & 2 & 0.4 \\
P. Multiple & 1 & 5 & 12 & 3 & 53.1 \\
Binary A. & 2 & 4 & $(6,8)$ & $(4,2)$ & $(0.6,1.8)$ \\
Arithmetics & 4 & 8 & $(20,8,3,4)$ & $(10,4,1,4)$ & $(10.3,3.7$ \\
& & & & & $3.5,14.6)$ \\
E. Grammar & 3 & 10 & $(6,3,3)$ & $(2,1,1)$ & $(1.4,0.3,1.9)$
\end{tabular}

Table 1: Generation task results.

The Generation results show that non-terminal symbols are used with two aims: i) abstracting a set of terminal symbols, e.g. the first procedure of the Arithmetics domain (with 20 lines, learned from 10 strings in 10.3 seconds) processes any digit in the set $\{0, \ldots, 9\}$; ii) grouping multiple rules, e.g. in English Grammar one procedure represents a Noun Phrase $(N P)$ that is composed of one or more adjectives $(a)$ and a noun $(n)$, so it computes the rules $N P \rightarrow$ an $\mid a N P$.

Table 2 shows the results for the Recognition tasks. In these experiments the CFGs grammars are given so we explore the performance of our approach using larger stack sizes. For each domain we report the size of the stack (which limits the max depth of the possible parse trees), the number of strings, and the total planning time required for parsing the strings.

\begin{tabular}{lrrr} 
& Stack & Strings & Time(s) \\
\hline AnBn & 51 & 1 & 70.67 \\
Parenthesis & 52 & 1 & 19.29 \\
P. Multiple & 52 & 1 & 129.19 \\
Binary A. & 15 & 2 & 62.87 \\
Arithmetics & 25 & 4 & 137.76 \\
E. Grammar & 92 & 1 & 325.44
\end{tabular}

Table 2: Recognition task results.

\section{Related work}

The learning of CFGs can also be understood in terms of activity recognition, such that the library of activities is formalized as a CFG, the library is initially unknown, and the input strings encode observations of the activities to recognize. Activity recognition is traditionally considered independent of the research done on automated planning, using handcrafted libraries of activities and specific algorithms [Ravi et al., 2005]. An exception is the work by Ramírez and Geffner [2009; 2010] where goal recognition is formulated and solved with planning. As far as we know our work is the first that tightly integrates the tasks of (1) grammar learning, (2) recognition and (3) production using a common planning model and an off-the-shelf classical planner.

Hierarchical Task Networks (HTNs) is a powerful formalism for representing libraries of plans [Nau et al., 2003]. HTNs are also defined at several levels such that the tasks at one level are decomposed into other tasks at lower levels with HTN decomposition methods sharing similarities with production rules in CFGs. There is previous work in generating HTNs [Hogg et al., 2008; Lotinac and Jonsson, 2016] and an interesting research direction is to extend our approach for computing HTNs from flat sequences of actions. This aim is related to Inductive Logic Programming (ILP) [Muggleton, 1999] that learns logic programs from examples. Unlike logic programs (or HTNs) the CFGs that we generate are propositional and do not include variables. Techniques for learning high level state features that include variables are promising for learning lifted grammars [Lotinac et al., 2016].

\section{Conclusions}

There is exhaustive previous work on learning CFGs given a corpus of correctly parsed input strings [Sakakibara, 1992; Langley and Stromsten, 2000] or using positive and negative examples [De la Higuera, 2010; Muggleton et al., 2014]. This work addresses generating CFGs using only a small set of positive examples (in some cases even one single string that belongs to the language). Furthermore we follow a compilation approach that benefits straightforwardly from research advances in classical planning and that is also suitable for production and recognition tasks with arbitrary CFGs.

Our compilation bounds the number of rules $m$, the length of these rules $n$, the size of the stack $\ell$ and the length of the input strings $z$. If these bounds are too small, the classical planner used to solve the output planning task will not be able to find a solution. Larger values for these bounds do not formally affect to our approach, but in practice, the performance of classical planners is sensitive to the size of the input. Interestingly our approach can also follow an incremental strategy where we generate the CFG for a given sub-language and then encode this sub-grammar as an auxiliary procedure for generating more challenging CFGs [Segovia-Aguas et al., 2016b].

The size of the compilation output also depends on the number of examples. Empirical results show that our approach is able to generate non-trivial CFGs from very small data sets. Another interesting extension would be to add negative input strings, which would require a mechanism for validating that a given CFG does not generate a given string, or to accept incomplete input strings that would require combining the generation and production mechanisms.

\section{Acknowledgements}

This work is partially supported by grant TIN2015-67959 and the Maria de Maeztu Units of Excellence Programme MDM2015-0502, MEC, Spain. 


\section{References}

[Alford et al., 2009] Ronald Alford, Ugur Kuter, and Dana S Nau. Translating htns to pddl: A small amount of domain knowledge can go a long way. In IJCAI, pages 1629-1634, 2009.

[Baier et al., 2007] Jorge A Baier, Christian Fritz, and Sheila A McIlraith. Exploiting procedural domain control knowledge in state-of-the-art planners. In ICAPS, pages 26-33, 2007.

[Bonet et al., 2010] Blai Bonet, Hector Palacios, and Hector Geffner. Automatic derivation of finite-state machines for behavior control. In AAAI Conference on Artificial Intelligence, 2010.

[Chomsky, 2002] Noam Chomsky. Syntactic structures. Walter de Gruyter, 2002.

[De la Higuera, 2010] Colin De la Higuera. Grammatical inference: learning automata and grammars. Cambridge University Press, 2010.

[Gold, 1967] E Mark Gold. Language identification in the limit. Information and control, 10(5):447-474, 1967.

[Helmert, 2006] Malte Helmert. The Fast Downward Planning System. Journal of Artificial Intelligence Research, 26:191-246, 2006.

[Hogg et al., 2008] Chad Hogg, Héctor Munoz-Avila, and Ugur Kuter. Htn-maker: Learning htns with minimal additional knowledge engineering required. In $A A A I$, pages 950-956, 2008.

[Jiménez and Jonsson, 2015] Sergio Jiménez and Anders Jonsson. Computing Plans with Control Flow and Procedures Using a Classical Planner. In Proceedings of the Eighth Annual Symposium on Combinatorial Search, SOCS-15, pages 62-69, 2015.

[Koller and Hoffmann, 2010] Alexander Koller and Jörg Hoffmann. Waking up a sleeping rabbit: On naturallanguage sentence generation with ff. In ICAPS, pages 238-241, 2010.

[Langley and Stromsten, 2000] Pat Langley and Sean Stromsten. Learning context-free grammars with a simplicity bias. In European Conference on Machine Learning, pages 220-228. Springer, 2000.

[Lotinac and Jonsson, 2016] Damir Lotinac and Anders Jonsson. Constructing Hierarchical Task Models Using Invariance Analysis. In Proceedings of the 22nd European Conference on Artificial Intelligence (ECAI'16), 2016.

[Lotinac et al., 2016] Damir Lotinac, Javier Segovia, Sergio Jiménez, and Anders Jonsson. Automatic generation of high-level state features for generalized planning. In $I J$ CAI, 2016.

[Martín and Geffner, 2004] Mario Martín and Hector Geffner. Learning generalized policies from planning examples using concept languages. Appl. Intell, 20:9-19, 2004.
[Muggleton et al., 2014] Stephen H Muggleton, Dianhuan Lin, Niels Pahlavi, and Alireza Tamaddoni-Nezhad. Metainterpretive learning: application to grammatical inference. Machine learning, 94(1):25-49, 2014.

[Muggleton, 1999] Stephen Muggleton. Inductive logic programming: issues, results and the challenge of learning language in logic. Artificial Intelligence, 114(1):283-296, 1999.

[Nau et al., 2003] Dana S Nau, Tsz-Chiu Au, Okhtay Ilghami, Ugur Kuter, J William Murdock, Dan Wu, and Fusun Yaman. Shop2: An htn planning system. J. Artif. Intell. Res.(JAIR), 20:379-404, 2003.

[Palacios and Geffner, 2009] Héctor Palacios and Héctor Geffner. Compiling uncertainty away in conformant planning problems with bounded width. Journal of Artificial Intelligence Research, 35:623-675, 2009.

[Ramírez and Geffner, 2009] Miquel Ramírez and Hector Geffner. Plan recognition as planning. In IJCAI, pages 1778-1783, 2009.

[Ramırez and Geffner, 2010] Miquel Ramırez and Hector Geffner. Probabilistic plan recognition using off-the-shelf classical planners. In Proceedings of the Conference of the Association for the Advancement of Artificial Intelligence (AAAI 2010), pages 1121-1126, 2010.

[Ramirez and Geffner, 2016] Miquel Ramirez and Hector Geffner. Heuristics for planning, plan recognition and parsing. arXiv preprint arXiv:1605.05807, 2016.

[Ravi et al., 2005] Nishkam Ravi, Nikhil Dandekar, Preetham Mysore, and Michael L Littman. Activity recognition from accelerometer data. In $A A A I$, volume 5, pages 1541-1546, 2005.

[Sakakibara, 1992] Yasubumi Sakakibara. Efficient learning of context-free grammars from positive structural examples. Information and Computation, 97(1):23-60, 1992.

[Schwenger et al., 2016] Maximilian Schwenger, Alvaro Torralba, Jörg Hoffmann, David M Howcroft, and Vera Demberg. From openccg to ai planning: Detecting infeasible edges in sentence generation. In International Conference on Computational Linguistics, 2016.

[Segovia-Aguas et al., 2016a] Javier Segovia-Aguas, Sergio Jiménez, and Anders Jonsson. Generalized planning with procedural domain control knowledge. In ICAPS, 2016.

[Segovia-Aguas et al., 2016b] Javier Segovia-Aguas, Sergio Jiménez, and Anders Jonsson. Hierarchical finite state controllers for generalized planning. In IJCAI, 2016.

[Vallati et al., 2015] Mauro Vallati, Lukáš Chrpa, Marek Grzes, Thomas L McCluskey, Mark Roberts, and Scott Sanner. The 2014 international planning competition: Progress and trends. AI Magazine, 36(3):90-98, 2015.

[Winner and Veloso, 2003] Elly Winner and Manuela Veloso. Distill: Learning domain-specific planners by example. In ICML, pages 800-807, 2003. 